4th International Conference Photoinduced Phase Transitions and Cooperative Phenomena, Wrocław 2011

\title{
PIPT from the Beginning to Future
}

\author{
H. Cailleau ${ }^{a, b, *}$, T. Luty ${ }^{c}$, S. Koshihara $^{b}$, M. Servol $^{a}$, M. Lorenc $^{a}$, \\ M. Buron-Le Cointe ${ }^{a}$ And E. COllet ${ }^{a}$
}

\begin{abstract}
${ }^{a}$ Institut de Physique de Rennes, Université de Rennes 1-CNRS, UMR 6251, Rennes, France
${ }^{b}$ JST, CREST and Department of Material Science, Tokyo Institute of Technology, Tokyo, Japan ${ }^{c}$ Institute of Physical and Theoretical Chemistry, Wrocław University of Technology, Wrocław, Poland

The birth of the field of photoinduced phase transitions was strongly influenced by the conceptual viewpoint expressed by Professor Y. Toyozawa on the condensation of relaxed excitons. Since this first period, twenty years ago, this young field has been expanding rapidly along a diversity of directions. Nowadays, it goes hand in hand with the challenges of today's science: emergence, nonlinearity, coherence, far away from equilibrium, for example. The control of the functionality of a material via photoexcited states poses many new fundamental questions. Some of them will be overviewed: (i) the nature of the control parameters and the nature of the relevant collective variables, especially the order parameters, which characterize the evolution of the system, (ii) the difference between photoinduced transformations under continuous light irradiation and those resulting from an ultrashort laser pulse, (iii) the physical mechanisms of ultrafast photoinduced phase transitions from the formation and proliferation of phototransformed entities to the softening of a collective mode.
\end{abstract}

PACS: 64.60.-i, 64.70.K-, 78.47.D-

\section{Flash-back}

This paper is dedicated in memoriam of Professor Yutaka Toyozawa, a founding father of the field of photoinduced phase transitions (PIPT). After the pioneering experimental work of Koshihara on the observation of the photoinduced neutral-to-ionic instability [1], the paper of Prof. Y. Toyozawa, "Condensation of relaxed excitons in static and dynamic phase transitions" [2], is the fingerprint of his inspiring contribution. He brought out some conceptual keys which strongly influenced our research and that of many of our colleagues. In the conclusion of this paper he emphasizes "the usefulness of the global view-point ... considering the crossover of the excited and the ground electronic states and correlating static and dynamic phase transitions through the motion of (spontaneously or optically) condensed elementary excitations".

A second step was the Taniguchi symposium in 1996 "Relaxations of excited states and photoinduced phase transitions" chaired by Prof. Nasu [3]. The aim of this symposium was to discuss the new problem of unconventionally photoactive materials. In these materials the relaxation of optically excited states result in collective motions or drastic structural changes, involving a large number of atoms and electrons. Actually, in a way this symposium was the precursor of the PIPT conferences. The extension of the physics of self-trapped excitons, which Toyozawa has largely contributed to [4], to systems with electron-spin-lattice cooperative interactions was discussed from the viewpoint of both material science and solid state spectroscopy.

* corresponding author; e-mail:

herve.cailleau@univ-rennes1.fr
Throughout this first period different theoretical papers were published, developing this approach to establish some backgrounds in the understanding of photoinduced phase transitions $[3,5,6]$. At the same period, different approaches of photoinduced phase transitions were introduced [7], in particular to the photoinduced phonon instability in conventional semiconductors [8].

For twenty years now this emerging field has been expanding rapidly along diverse directions: correlated electrons systems, charge density waves, ultrafast magnetism, purely electronic phase transitions, chemical bonding, molecular switching in materials, soft matter, etc. In parallel to the development of theoretical models [9], more and more elaborated ultrafast optical spectroscopic experiments have been conducted on a diversity of materials. These experiments gave the opportunity to observe different kinds of ultrafast dynamical processes during the first stages of photoinduced transformations, mainly in correlated electron systems, as illustrated in previous PIPT conferences $[10,11]$ and different reviews $[12,13]$. Moreover, new experimental tools have appeared, such as fast and ultrafast time-resolved X-ray and electron diffraction [14] or spectroscopic application of intense terahertz $(\mathrm{THz})$ pulse [15-17]. At present this field is at the frontier of science as discussed in the following Sect. 2. It raises more and more exciting questions/ challenges in both experimental and theoretical research. Some of them will be overviewed and discussed in Sect. 3 . These will focus on electron-lattice systems in the continuation of the pioneering work of Toyozawa.

\section{Hand in hand with today's science}

Today, a current challenge in science and technology is not only to observe matter on ever smaller scale but also to direct its functionality at the relevant length, time 
and energy scales. A recent report from the Basic Energy Sciences Advisory Committee in USA, "Directing Matter and Energy: Five Challenges for Science and the Imagination", emphasizes this advent of control science [18]. A well known example is given by spectacular advances in nanoscience with the mastering of energy and information on the nanometer scale. Less hyped but none the less important is the tracking and control of dynamic processes in matter on ultrashort time scale. This feat can be accomplished thanks to the increase of sophisticated technologies and instrumentation in ultrafast science, giving fascinating capabilities to observe and understand not only the first stages of dynamic processes in atoms, molecules or materials but also to study how matter works and can be directed to a desired outcome. A nice example is provided by femtochemistry, a field awarded Nobel Prize in 1999 [19]. In the photoexcited electronic state the interatomic potential changes and determines the motion of atomic wave packets prepared by a femtosecond light pulse. This may induce drastic effects such as breaking of bonds and subsequent chemical transformation from transient states. Arguably, the most spectacular advance is the ability to decide on the outcome of a chemical reaction through coherent control of transformation pathway. More recently the new field of PIPT has emerged within material science, which deals with phase transition upon absorption of light. Thus an ultrashort laser pulse may induce the transformation of the material towards another phase of different electronic and/or structural order. This triggers drastic switching of the macroscopic physical properties of materials, for example from non-magnetic to magnetic or from insulator to conductor. The goal is then to realize at the level of a material which has been achieved at the level of a molecule in femtochemistry. This corresponds to a control of the functionality of a material via electronic excited states and not by acting on the ground state as commonly done.

Photoinduced phase transitions driven by an ultrashort laser pulse constitute a new step in the advent of emergent phenomena and the control of complexity in materials science. Complexity is an ambiguous word but a simple definition can be based on the occurrence of emergent phenomena [20]. Complex phenomena take place

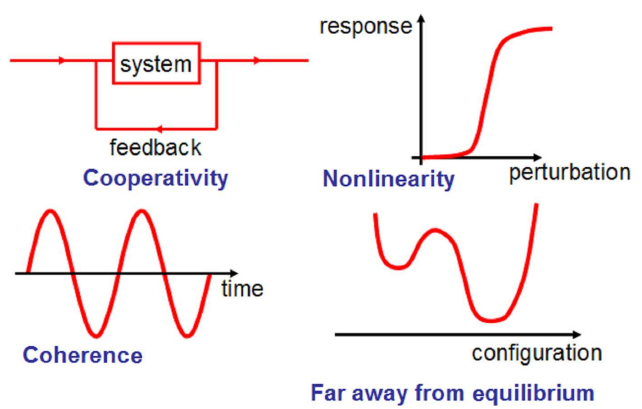

Fig. 1. Schematic pictures of interconnected key issues of PIPT. in a system composed of many interacting constituents whereby the collective properties of the whole are more than the sum of their individual behaviour. Thus, PIPT illustrate anew the famous Anderson's words: "More is different" [21]. Along this line, let us also note that, among the four grand challenges in physics revealed by a survey of researchers in UK by the Engineering and Physical Sciences Research Council, one is "emergence and physics far from equilibrium" [22].

Thus, the physical background of PIPT combines different interconnected key issues which are at the heart of grand challenges in today's science, as schematically drawn in Fig. 1.

\subsection{Cooperativity and nonlinearity}

Cooperative interactions between constituents of a complex system drive the emergence of remarkable properties of matter such as phase transitions at thermal equilibrium. Such cooperative interactions are also of great significance for the out-of-equilibrium phase transformations. In particular, this is the case for photoinduced phase transitions triggered by an ultrashort laser pulse, to an extent that goes beyond the femtochemistry of independent molecules. Namely, the medium is no more passive but active through positive feedback. This gives rise to an effective field acting on each atom or molecule, which is at the origin of strong nonlinear responsiveness to light perturbation. A linear system is divided into parts which can be solved separately and finally recombined, such as normal modes for the states of vibrations. In this sense, a linear system is precisely equal to the sum of its parts. But whenever parts of a system cooperate, nonlinear response and emergent phenomena come into life, and some complexity is introduced. Thus, the simultaneous absorption of a huge number of photons induces more than the sum over individual events. In particular, this is manifested by self-amplification and threshold effect in the response of the system to strong external perturbations, like intense light pulse.

\subsection{Coherence}

Manipulating coherence is a fascinating adventure in science, from laser light to ultracold quantum degenerate atomic gases. The collective wave fields vibrate in unison, in phase, and interfere. They become so efficient that they drive spectacular physical properties. In femtochemistry, an ultrashort light pulse prepares a coherent atomic wave packet for every photoexcited molecule. The same coherent process may occur in materials but in this case it concerns collective degrees of freedom. This is the case of the generation of coherent optical phonons by light excitation, corresponding to ultrafast in-phase oscillations of a long range structural order at macroscopic scale. These can be revealed by oscillation of the Bragg peak intensities [23]. This is fundamentally different from the Debye-Waller effect and thermal diffuse scattering which result from thermally excited incoherent 
phonons. In addition, in case of localized molecular excitations in solid state coherent nucleation and growth processes can take place [24]. These different dynamics are no more stochastic as at thermal equilibrium but instead become deterministic and ultrafast. The laser producing an ultrashort light pulse is no longer a mere heat source. Instead it coherently controls the material by directing the system along the desired pathways on the potential energy landscape. Mastering coherent processes is a big challenge.

\subsection{Far away from equilibrium}

Characterizing and understanding matter far away from equilibrium constitute a fundamental challenge in science since our current understanding of matter relies profoundly on the concept of equilibrium, enough to mention the universal success of thermodynamics. Photoinduced phase transitions bring about new kind of out-of-equilibrium phenomena of which the outcome is not the recovery of the thermal equilibrium state but rather forcing a new macroscopic state far away from equilibrium. Actually different subsystems of different nature play their part, such as electrons, spins, phonons, molecular configurations, unit cell deformation, etc. The challenge is to determine what are the relevant physical parameters to describe such cooperative non-equilibrium dynamics with a gigantic number of degrees of freedom, and what are the control parameters. In other words, the goal is to drastically reduce the number of variables from macroscopic to "handful". The present progress of ultrafast technologies, such as optical or X-ray and electron scattering techniques, gives a golden opportunity to observe in real-time the evolution of a system on its potential energy landscape and so to watch directly how matter rearranges. This allows determining the consecutive steps taking place for different kinds of degrees of freedom on different length and time scales, from deterministic coherent dynamics to stochastic thermal processes.

Let us note that the ultrafast photoinduced transformations arouses both academic and technological interests:

- experimental and theoretical research on the complex evolution of a macroscopic system from the initially prepared wave packets to the transformation of the material. These overcome frontiers between fields and shed new light on many fundamental questions in physics (quantum, statistical, condensed matter, non-equilibrium, nonlinear) in order to understand how matter works. We are faced with several new and challenging physical problems;

- impact on future technologies, for instance ultrafast information processing based on the control of light-driven switching of the functionality of a material, requiring the answer to key questions, as for instance: How to direct the system through a complex pathway from atomic to material scales?
What are the limits for transformation speed? How to design new materials and systems with desired outcome?

\section{Beholding the complex picture}

The field of photoinduced phase transitions remains very young and it is difficult to draw a global picture. This is particularly true in comparison with the case of phase transitions at thermal equilibrium. The key point for phase transitions at thermal equilibrium is that, despite their apparent dissimilarities illustrated by a large diversity of systems of different nature, interactions and properties, a universal description has been established: order parameter, the Landau description, critical phenomena, etc. Particularly demonstrative is the picture of the Landau expansion of thermodynamic potential, such as free energy, as a function of the order parameter for given values of control parameters, temperature, pressure, electric or magnetic field. The potential energy picture is a universal concept to describe the stability, metastability and instability of a system, and its subsequent dynamics or kinetics. This is well known in mechanics, thermodynamics and also chemical kinetics. Obviously there are some limits for such simple potential energy pictures, notably for the statistical physics of phase transitions since they are based on mean field approximation. Nevertheless they capture the gross physical features. Therefore, they remain very illustrative and productive, providing very useful guidelines for researchers. Such a universal description of photoinduced phase transitions has not been established yet and we are faced with a diversity of different experimental results [12, 13] and theoretical models [9]. It is exciting physics opening a huge space but along a twisting road of science. It is needed to extend existing theories to new regimes and to develop fundamentally new concepts. The universality for photoinduced phase transitions is probably weaker than it is at thermal equilibrium since it corresponds to an additional degree of complexity. However it is essential to try to catch some general guidelines to orient future research. In particular, in both thermal equilibrium and non-equilibrium a central point is to determine what are the nature of the control parameters and that of the relevant collective variables, in particular the order parameters, which characterize the evolution of the system.

In this Section we would like to discuss three main points. First, we provide some general considerations on long range order and symmetry change at phase transition which lays down powerful unifying framework for thinking generally about variety of orders in matter and can be extended to the case of photoinduced phase transitions. Second, we discuss how the physics is different between a photosteady bistability and a phase transition driven by an ultrashort laser pulse. Finally, we describe two limit mechanisms for the ultrafast dynamics of the transformation in this second situation, i.e. on the 
one hand, the formation and proliferation of phototransformed entities and, on the other hand, the softening of a collective mode.

\subsection{Long range order: symmetry-breaking or not?}

A phase is a homogeneous macroscopic part of matter exhibiting some electronic, magnetic and/or structural order. The fingerprint of order in matter, both on long range and short range, is inscribed in the behaviour of the relevant density function $\rho(\boldsymbol{r})$ in space: electronic, magnetic, structural... The scattering of waves (X-ray, electron, neutron) is the direct way to probe this density and so to watch atoms in matter. Where are they? What are they doing? The scattering pattern corresponds to a Fourier analysis of the related density. However, as it is the scattering intensity and not the amplitude which is measured, it actually probes the two-point densitydensity correlation function

$\langle\rho(\boldsymbol{r}) \rho(0)\rangle$.

At long distance this correlation function does not converge in the case of long range order. For example, it is periodic for crystalline order and quasiperiodic for incommensurably modulated solids, such as charge density wave systems. On the other hand, the correlation function converges towards a smaller constant value for short range order. As a rule, this decrease is characterized by a typical length, called the correlation length. In liquid or glassy state only short range order exists whereas in crystalline state long and short range orders coexist, as discussed below.

In a crystalline material the instantaneous density at time $t$ of the unit cell $n$ can be decomposed into two parts

$$
\rho_{n}(t)=\rho_{\text {cell }}(t)+\Delta \rho_{n}(t),
$$

where the first part $\rho_{\text {cell }}(t)$ corresponds to the average over all unit cells in the crystal and the second part $\Delta \rho_{n}(t)$ is the deviation of the given cell $n$. The symmetry of the long range order is included in the global density which corresponds to the periodic repetition in the crystal of the first part. The correlation function of the deviations between different cells may present only short range order. As a consequence of such decomposition, the scattering pattern is also decomposed between the Bragg peaks which probe only long range order, and diffuse scattering for the short range order. The example of a crystal at thermal equilibrium discussed in Sect. 2, i.e. the case of thermally excited incoherent phonons, provides a good illustration of this decomposition into two components, the Bragg peaks affected by the Debye-Waller factor and the thermal diffuse scattering.

Conventional scattering techniques probe the time average which is equal to the statistical ensemble average at thermal equilibrium. The same kind of conventional scattering techniques can be used in the case of a photostationary state, or a long-lived excited state once irradiation is stopped. The situation is different in the case of ultrafast scattering techniques used to directly observe structural dynamics. Indeed, today they are able to catch diffraction patterns with a time resolution shorter than the time scale of atomic motions [14]. Then, they can probe the quasi-instantaneous density $\rho(\boldsymbol{r}, t)$, and follow the time evolution of atomic motions and crystal transformation induced by an ultrashort laser pulse. However, the decomposition between an average over all cells in the crystal and local deviations remains valid. It is important to become aware of the fundamental difference between an instantaneous picture of a crystal at thermal equilibrium and that of the evolving state clocked after an initial excited state prepared by an ultrashort laser pulse. The difference between the instantaneous picture of thermally excited incoherent phonons and in-phase oscillations of coherent phonons, quoted in Sect. 2, is a good example (Fig. 2).

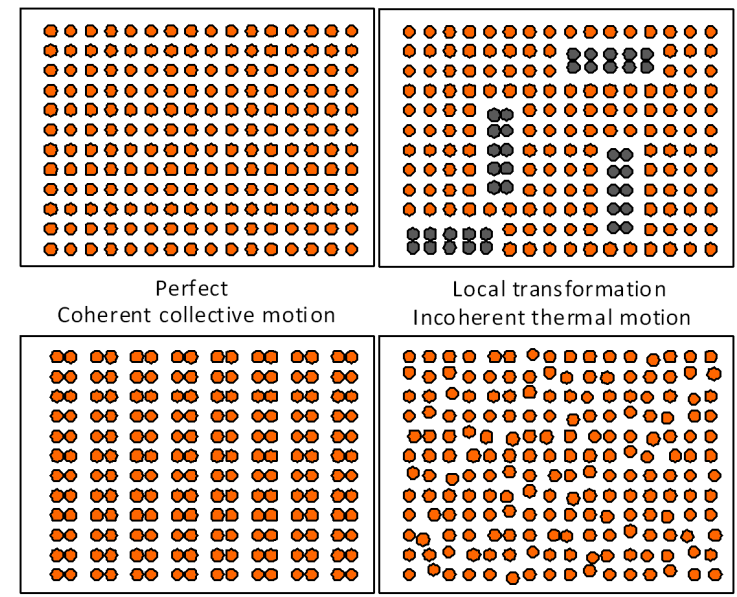

Fig. 2. Instantaneous pictures of a crystal exhibiting local transformation, coherent and incoherent phonons.

A phase transition is often related to a change of symmetry. Symmetry is one of the strong foundations of science. The symmetry breaking analysis and the concept of order parameter, introduced by Landau, are so universal that we can again apply them to photoinduced phase transitions, including those driven by an ultrashort laser pulse. This is also directly connected to the occurrence of emergent properties as discussed by Anderson [21]. The symmetry of an instantaneous state is implicitly included in the global density $\rho(, t)$ which describes the periodic repetition of $\rho_{\text {cell }}(t)$, the average over all unit cells. Within the Landau analysis it can be decomposed as

$$
\rho(\boldsymbol{r}, t)=\rho_{0}(\boldsymbol{r}, t)+\Delta \rho(\boldsymbol{r}, t),
$$

where $\rho_{0}$ is left invariant by the elements of the initial symmetry group $G_{0}$ of the high symmetry phase and $\Delta \rho$ is the change of density function to form the low symmetry phase, described by a symmetry group $G \subset G_{0}$. The method of analyzing symmetry variation, proposed by Landau, is based on expanding $\Delta \rho$ in terms of an irreducible representation (IR) of the initial group $G_{0}$ : 


$$
\Delta \rho(\boldsymbol{r}, t)=\sum_{i} \eta_{i}(t) \phi_{i}(\boldsymbol{r}),
$$

where the $\eta_{i}$ are the vector components of the order parameter $\eta$ of dimension equal to that of the IR and the $\phi_{i}(\boldsymbol{r})$ are the basis functions of the same IR [25]. As a matter of fact the above order parameter corresponds to an average over all unit cells and then characterizes the new long range order. In other words, the value of its modulus measures the degree of symmetry breaking. This can become very small when it is going to zero continuously on approaching a critical temperature at thermal equilibrium, i.e. in case of a second order phase transition. On the contrary, it presents a discontinuous evolution with a jump at the transition temperature for a first order phase transition. When the phase transition is associated with a change of the multiplicity of elementary unit cells, the symmetry breaking manifests itself by the appearance of superlattice Bragg reflections in the diffraction pattern. Their intensity is proportional to the modulus square of the order parameter.

For a phase transition at thermal equilibrium or a photostationary bistability the order parameter is homogeneous, i.e. constant in space (except the possibility of domains and domain walls related to the symmetry breaking). This is no more true for a laser pulse driven phase transition since the order parameter may vary in space due to a finite penetration depth of pump light. Indeed, the phototransformed material is no more homogeneous at the macroscopic scale along the direction parallel to the laser beam. The density of absorbed photons, which is a control parameter, varies along this direction, and can cross over a critical value. Therefore, the order parameter can be zero at some depth and non-zero at another. The evolution of the order parameter as a function of the depth can be discontinuous, first-order-like, or continuous, second-order-like. An interface separates the two subsequent coexisting phases. This is an obvious fact in the first situation. The second situation is also without ambiguity because symmetry exists or not and there is no other alternative. In addition, similar phenomena take place on the crystal surface in relation with the Gaussian shape of the laser beam (in general the experimental geometry is such that the laser beam is normal to the sample surface).

There exist phase transitions without change of symmetry, such as the Mott transition or spin transition, for example. They are generally called isostructural and are similar to the gas-liquid phase transition, with a phase diagram at thermal equilibrium composed of a first order line (coexistence line) ending at a critical point. Let us note that a large number of materials exhibiting photoinduced phase transitions are of this type. It is not possible to define an order parameter in the sense of symmetry breaking. But, it is known that the thermodynamics of the gas-liquid phase transition is similar to that of ferromagnetic one. Therefore, the discontinuity of the density along the first order line in the thermodynamic control parameter space (pressure-temperature) play the same role that the magnetization jump along the horizontal first order line in the magnetic field-temperature phase diagram for the ferro-to-paramagnetic phase transition. Actually this extends the concept of order parameter to a so-called totally symmetrical order parameter. However, situations where this totally symmetrical order parameter is coupled to another one describing a symmetry breaking also exist. This situation can be efficiently analysed in terms of the Landau theory [26].

In addition, there exist phase transitions with a change of symmetry but without group-subgroup relation. They are called reconstructive phase transitions and the graphite-diamond is a good example. The photoinduced cooperative transformation observed in graphite illustrates this situation from within the PIPT field. Finally, let us note that a locking phase transition, such as occurring in charge density wave systems from an incommensurate phase to a commensurate one, is of another nature. In fact it corresponds to a change of the critical wave vector.

Emergent collective behaviour results from the symmetry breaking, giving rise to rigidity. Indeed, any change of the order parameter, such as its orientation or its spatial variation, costs energy [27]. It is appropriate to distinguish between discrete symmetry breaking (sharp domain wall) and continuous symmetry breaking (soft elementary excitations, such as acoustic phonons in crystals, magnons in ferromagnets, etc.). A particular situation of continuous symmetry breaking is that of incommensurably modulated crystal [28]. The free energy is well invariant with respect to changes in the relative position of modulation and basic structure, but not the Hamiltonian once the anharmonicity is taken into account. Then, phasons which are associated with the phase modulation of the incommensurate order parameter are always diffusive in the long wavelengths limit, contrary to acoustic phonons and magnons. It is surely important to consider all these features in the collective dynamics of ultrafast photoinduced phase transitions, giving rise to challenging experiments. Let us note that such a situation of collective dynamics with a broken symmetry was recently studied in a charge density wave system [29, 30].

\subsection{Photosteady vs. light pulse driven}

Physics is different between the two kinds of photoinduced phase transitions: bistability between photosteady states or light pulse driven phase transition. The physical effects are different albeit the irradiating fluence is the same, i.e. for the same number of absorbed photons per second. When the duration of the laser pulse is shorter than the time scale of atomic motions, non-thermal photoinduced phenomena occur at first.

In the first case, under continuous photoirradiation, some systems, such as spin crossover [31], cyano-bridged metal [32], metal oxide [33] and even biochemical ones [34], may exhibit light driven bistability between two macroscopic states. A general universal frame exists within the physics of nonlinear dynamics and bifurcations [35]. This is based on a powerful geometric 
approach for analysing the stability of a dynamical system, which emphasizes qualitative rather than quantitative questions. In some way, this scientific approach presents similarity with the Landau analysis at thermal equilibrium. In the case of photosteady bistability, the flow of energy from photon flux competes with the dissipation in the thermal bath. The cooperative interactions lead to a feedback mechanism and so to nonlinear kinetic law for the thermally activated relaxation process. The kinetic coefficients $k$ are no more constant but depend on the state of the active medium. Thus, within a mean field approach they can be simply expressed as a function of the concentration $x$ of phototransformed entities. For instance, we will discuss the case of the bistability of spin crossover crystal between a low-spin (LS) and a high-spin (HS) state. At low temperature $k_{\mathrm{LS} \rightarrow \mathrm{HS}} \ll k_{\mathrm{HS} \rightarrow \mathrm{LS}}$ and only the second kinetic coefficient can be taken into account. It can be written

$$
k(x)=k_{0} \exp (-a x),
$$

where $x$ is the concentration of HS molecules and $a$ is a coefficient dependent on the temperature which is the control parameter fixed by the thermal bath [36-38]. Within the frame of mean field approximation, the set of master equation is reduced to a simple one-dimensional phenomenological kinetic equation which expresses the competition between the gain term due to photon flux and the loss term due to dissipation in the thermal bath

$$
\mathrm{d} x / \mathrm{d} t=\alpha I(1-x)-k(x) x,
$$

where $I$ is the light intensity which is the other control parameter in addition to temperature $T$, and $\alpha$ the light-conversion coefficient from LS to HS states. This equation can be solved graphically to find the fixed points corresponding to the equilibrium (steady) solutions. Depending on the values of control parameters, $T$ and $I$, there are one, two or three intersection points. In the case of three points, these ones correspond to respectively two stable (regression of small disturbances) and one unstable (growing of small disturbances) equilibrium states. This is again more illustrative if we use a potential picture. Indeed, such one-dimensional dynamical equation can be written in terms of the derivative of a dynamical potential $V(x)$ :

$$
\mathrm{d} x / \mathrm{d} t=-\mathrm{d} V(x) / \mathrm{d} x .
$$

The dynamical potential must not be confused with a thermodynamic potential. For instance, at low temperature the thermodynamic potential presents only a stable equilibrium point, the low spin state, while the dynamical potential may present two stable equilibrium points (bistable regime between LS and HS state) (Fig. 3). One of these points is less stable (metastable) with a higher potential. The behaviour of this dynamical potential governs the nonequilibrium dynamical phase transition. A bifurcation-phase diagram in the control parameter space $(I, T)$ may be obtained, which corresponds to the line where the two equilibrium points are equally stable and which ends at one critical point (Fig. 3). There are also two lines associated with the limits of metastability which are the bifurcation lines. This is very analogous with the gas-liquid phase transition and show that in the situation of photosteady bistability we find a universality similar to the one at thermal equilibrium. In the language of catastrophe theory this corresponds to a cusp catastrophe [35].

Furthermore, additional symmetry change may take place in the photosteady state which are not observed at thermal equilibrium but can only be attainable under non-equilibrium condition by light irradiation. Thus, some examples of such photosteady orders have been observed in some materials, such as a ferromagnetic order at low temperature (photoinduced magnetic symmetry breaking) [39] or complex structural HS orders with new cell multiplicities [40].
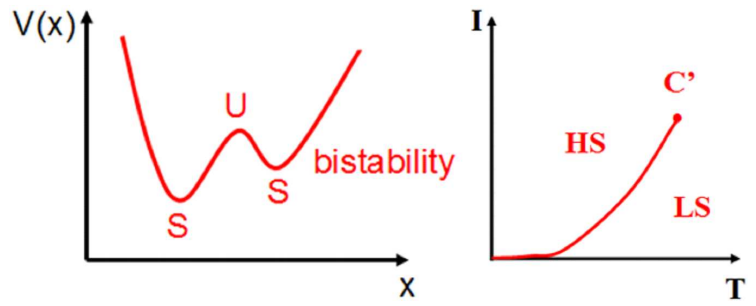

Fig. 3. Dynamical potential and bifurcation-phase diagram for a photosteady bistability [40].

A universal description is thus well established in the case of a photosteady bistability, with a deep analogy with that of phase transitions at thermal equilibrium. The situation is less clear for ultrafast phase transition driven by a laser pulse. A key point is that in materials different subsystems of a different nature (electrons, spins, phonons, cell deformations, etc.) play their part on time and length scales which are often significantly different. This is typical of multiscale phenomena.

The description of behaviour on one scale uses information from other scales. On each of them a particular approach has to be used. On a given observational time scale characteristic of the dynamical evolution of certain degrees of freedom, for other degrees of freedom some are faster and act by their quantum or statistical average, while some are slower and then frozen.

For instance, on the time scale of atomic oscillations, electronic degrees of freedom are averaged, whereas crystal deformation does not have time to occur. This bottleneck in the phase space may lead to a decoupling between the different sub-parts (electrons, phonons, cell deformation, etc.) which step in the relevant dynamical picture of the transformation. Therefore, there is not a unique dynamical description valid at every time scales, not a unique equation to describe the time evolution, not a unique potential energy landscape. For a given step, a particular potential energy has to be used to describe the evolution towards a quasi-equilibrium. This follows the Feynman's definition of equilibrium as being "when 
all the fast things have happened but the slow things have not" [41]. The quasi-equilibrium state is determined by relevant control parameters.

For instance, during the first steps the volume is fixed and then it acts as a control parameter. In addition, some photoinduced entities can be locally frozen during short time scales, in particular due to an energy barrier between molecular states [42], and a kind of glassy state is transiently observed, in other words a kind of broken ergodicity for the corresponding degrees of freedom [43].

Furthermore, the system is initially prepared in an excited electronic state by the intense laser pulse and when the pulse duration is shorter than the typical time scale of atomic motions, coherent structural dynamics can take place. This is a deterministic dynamics, not a stochastic kinetics described by a master equation, in other words a non-thermal process.

Let us notice that the separation of time scales plays its role both in quantum mechanics, as illustrated by the Born-Oppenheimer approximation, and in thermodynamics with the concept of quasi-equilibrium [44]. Therefore, depending on the given time scale, the potential energy picture goes from mechanical potential directing deterministic dynamics to thermodynamic potential for stochastic kinetics. The classical dynamics of an overdamped oscillator provides an illustrative analogy. Indeed, regardless of initial conditions, first a rapid initial transient dynamics governed by a second-order equation takes place, and only afterwards it behaves like a first-order one [35]. The situation becomes again more complex when it is difficult to unambiguously define distinct time scales for the different subsystems. Besides, the observation of the first deterministic steps of the material transformation is possible with ultrafast measurements now sampling with intervals shorter than the stochastization time.

\subsection{Nanodomains vs. softening}

Let us discuss the dynamical mechanisms driving the evolution of the material to form a photoinduced phase transition. Again the physical mechanisms are different between the formation of a new photosteady phase and an ultrafast transformation driven by a light pulse. Regarding the first case, a wide variety of methods, concepts and models exist to describe the pattern formation and dynamics in sustained non-equilibrium systems [45, 46]. They begin to be applied to the spatiotemporal structure and dynamics of photosteady bistabilities [47]. In the second case the dynamical processes present a supplementary degree of complexity.

Firstly, this is a multiscale phenomenon, on significantly different time scales which manifests itself successively on micro-, meso- and macroscales.

Secondly, a deterministic coherent dynamics and not stochastic process takes place during the first stages. A diversity of dynamical phenomena has been observed and a number of theoretical models have been studied. It is very difficult to draw a general picture. Only simple considerations are discussed below in two limit situations.
In a similar way to femtochemistry, the ultrashort laser pulse prepares a coherent atomic wave packet in the electronic excited state. Afterwards, this determines wave packet evolution which can drive in-phase oscillations and even change of state. For such spectacular phototransformation it is convenient to distinguish between different cooperative mechanisms, from the proliferation of nanodomains of localized phototransformed entities to the softening of a collective delocalized mode extended over entire crystal. These constitute the two limit situations, the first one follows the global viewpoint expressed by Toyozawa [2], the second one follows the concept of photoinduced phonon instability in semiconductors already introduced in Sect. 1 [8]. As the photon energy $h \nu$ is much greater than the thermal energy $k_{\mathrm{B}} T$, the photoinduced process allows attaining highly excited states. Therefore, the nature of photoinduced precursor dynamics can differ significantly from that of pretransitional phenomena at thermal equilibrium. For instance, the pretransitional dynamics at thermal equilibrium can be governed by a soft mode while the self-trapped excitons play a central role for the photoinduced precursor phenomena. In addition, the high energy of absorbed photons gives the possibility to transform the material to a phase not attainable at thermal equilibrium, i.e. a hidden phase [48, 49]. This is also true in the case of photosteady phases as discussed in Sect. 3.2 [39, 40].

Let us begin by discussing the first situation which concerns the formation and the proliferation of localized phototransformed entities (Fig. 4). The early stage of such photoinduced processes is directly related to the self-localization of an exciton in an insulating crystal, which has been theoretically investigated in detail by Toyozawa [4]. Inside this issue, Nasu reviews this point and clarifies the physical significance of the self-localization process due to lattice relaxation. The initial photoexcited electronic Bloch wave state, extending over entire crystal, spontaneously shifts towards a new self-localized excited state, implying large structural distortions only around it. Actually, this corresponds to the gradual disappearance of the spatial inter-site quantum coherence. It may proceed at the scale of a molecule, as for instance in spin-crossover crystal, but it may also involve a certain number of electrons and atoms or molecules. In this last case, a nanodomain with a new electronic and structural state has been prepared. This may imply the formation of polar entities as for the neutral-to-ionic phase transition. However, this occurs only locally at the nanoscale and there is no global symmetry breaking of the spatial parity.

The most direct observation of such nano-objects, with an internal structural coherence but incoherently distributed in the crystal, is realized through time-resolved diffuse scattering [50]. These phototransformed entities may cooperatively interact with the atoms/molecules in the surroundings. This may trigger coherent nucleation and growth [24, 51] and coherent phonons [52, 53]. This may even lead to the spontaneous formation of 

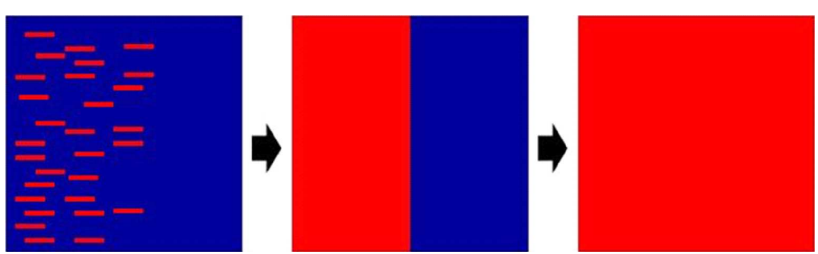

Fig. 4. Schematic illustration of the formation and proliferation of phototransformed entities.

a macroscopic domain in a second stage, with a new long range electronic and structural order. This is really the time when the photoinduced phase transition takes place, since long range order establishes only at this stage. Let us underline that this second step takes place at the mesoscale, concerning local equilibrium state in relation with the inhomogeneous nature of the distribution of phototransformed nanodomains originating from the shape and penetration depth of laser beam. The mechanisms are not yet well established and they are certainly diverse. It will be important, and not always easy, to disentangle coherent and thermal processes at this stage.

Furthermore, the response becomes nonlinear: the converted fraction as function of the excitation density shows that self-amplification takes place and threshold effect in the response to excitation density may occur [52-55]. An exciting challenge is the study of coherent pattern formation. This introduces a new kind of nonlinear spatiotemporal dynamics with regard to the purely stochastic one in the formation of steady states (cf. above). Recent results, in particular those presented during this PIPT4 conference, illustrate the diversity of such phenomena in different spin-charge-lattice coupled systems, from molecular to oxide materials, from the dynamics of charge density wave to the "Mottness" of electronic order, including light-induced superconductivity [56-67].

In the second situation concerning the softening of a collective mode we have to consider delocalized electronic excited states. After the ultrafast electronic thermalization following the photoexcitation of a huge number of electron-hole pairs, the new statistical average of the electronic distribution (the Fermi-Dirac) determines the change of interatomic forces. This induces a deterministic collective atomic oscillation around new equilibrium positions. Then a new relevant potential can be defined, constituting an extension of the Born-Oppenheimer approximation.

The collective oscillation mode is purely mechanical and the subsequent dynamical process is at constant entropy $[8,68]$. This behaviour is analogous to the oscillation of an adiabatic piston when the applied force compressing a gas is suddenly removed, assuming a small friction. The relevant potential energy is that of the electronic energy at constant entropy as a function of the normal coordinate of the lattice mode. For a high density of photoexcited electrons nonlinear effects occur. This can lead to the softening of the mode towards a cusp point bifurcation - phase transition, and so to a reverse symmetry breaking [68-70]. According to Landau's descrip- tion, the relevant collective normal coordinate is always the order parameter. However, the control parameter is no longer the temperature but the density $n$ of photoexcited electrons with a critical value $n_{\mathrm{c}}$ (cf. Fig. 5). During the early stage, the state of the material corresponds to an oscillating state of a long range atomic order. This is an unusual ordered state of matter. On approaching the critical concentration, the oscillation becomes more and more nonlinear. This is illustrated by the fact that the period is different from that of small oscillations. In the same way, the time averaged order parameter differs from the normal coordinate at the energy minimum.

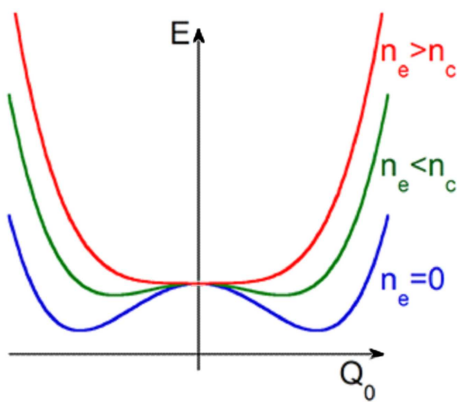

Fig. 5. Schematic picture of the energy potential governing the atomic motions for the case of delocalized electronic excited state: electronic energy as a function of the collective normal coordinate $Q_{0}$ (order parameter) shown for three different densities $n_{\mathrm{e}}$ of photoexcited electrons (control parameter) below and above the critical density $n_{\mathrm{c}}$.

Another key feature of ultrafast photoinduced phenomena is the dynamical process of volume expansion and other crystal deformations (shear, etc.). These concern macroscopic dimensions. They require a movement of the atoms or molecules over long distances. Actually, this process involves propagation of cell deformations limited by a speed similar to the sound velocity $[56,71]$. For instance, this translates to nanosecond range for a distance of a few $\mu \mathrm{m}$. Before this characteristic time scale, the crystal does not deform, despite local deformations due to the lattice self-trapping, and its initial volume is a macroscopic control parameter, while the pressure increases within the crystal. After the volume relaxation, i.e. when the deformation of unit cells has efficiently propagated over the crystal and that both for the parallel and perpendicular directions to the surface irradiated by the laser beam, the pressure within the crystal is in equilibrium with the external pressure. Therefore, it is the pressure which becomes the new control parameter. The crystal can be considered as a continuum medium and the deformation is governed by propagating nonlinear waves (shock waves) [72, 73], where the relevant collective variable (as order parameter field) and the crystal deformations are coupled. Their control defines new challenges. Moreover, the thermal effects due to slow heat diffusion through the sample are also to be taken into account at longer time scale $[57,74]$. 


\section{Concluding remarks}

This brief overview of some exciting questions at the heart of this young field of photoinduced phase transitions was an attempt to bring out some very general and simple ideas/concepts in memory of the pioneering work of Professor Toyozawa. At thermal equilibrium, the universal description of the transformation of matter between different organized forms at the macroscopic scale (phases) presents two Janus faces, entropy (Boltzmann) and symmetry (Landau). The extension to photoinduced phase transitions opens new challenges, since we are face to face with essentially non-independent, nonlinear, non-classical and non-equilibrium processes. Thus, the formation of a new phase triggered by an ultrashort laser pulse is a multiscale process. It is also governed by a deterministic coherent dynamics in early stages.

We are yet far from establishing a universal description of such challenging processes. However, we should remember that a general way of thinking is to discuss the behaviour of the system in terms of control parameters and relevant collective variables, in particular the order parameter, which characterize the evolution of the system. This overview focused mainly on electron-lattice systems, and not directly on other systems such as, for instance, magnetic materials or soft matter.

Even if a number of general considerations discussed here can be also applied to these situations, it is useful to refer the reader to recent reviews on the light manipulation of magnetic order [75] or soft and biochemical matter [34]. Another kind of cooperative photoinduced phenomena, not discussed here, concerns the photostationary macroscopic state originating from the quantum condensation of electron-hole-photon systems [76].

\section{Acknowledgments}

This work was supported by the Institut Universitaire de France, ANR (ANR-09-BLAN-0212), Europe (FEDER), Région Bretagne (CREATE 4146) and Rennes Métropole. H.C. would like to thank particularly the Tokyo Institute of Technology for its support during the writing of this paper.

\section{References}

[1] S. Koshihara, Y. Tokura, T. Mitani, G. Saito, T. Koda, Phys. Rev. B 42, 6853 (1990).

[2] Y. Toyozawa, Solid State Commun. 84, 255 (1992).

[3] Relaxation of Excited State and Photo-Induced Phase Transitions, Ed. K. Nasu, Springer, Berlin Heidelberg 1997.

[4] Y. Toyozawa, Optical Processes in Solids, Cambridge University Press, Cambridge 2003.

[5] E. Hanamura, N. Nagaosa, J. Phys. Soc. Jpn. 56, 2080 (1987).

[6] N. Nagaosa, T. Ogawa, Phys. Rev. B 39, 4472 (1989).

[7] A.L. Semenov, J. Exp. Theor. Phys. 87, 764 (1998).

[8] P. Stampfli, K.H. Bennemann, Phys. Rev. B 42, 7163 (1990).

[9] K. Yonemitsu, K. Nasu, Phys. Rep. 465, 1 (2008).
[10] The Second International Conference on Photo-induced Phase Transitions, Eds. M. Buron, E. Collet, J. Phys., Conf. Series 21, (2005).

[11] The LXII Yamada Conference on Photo-induced Phase Transition and Cooperative Phenomena (PIPT3), Eds. K. Tanaka, T. Ogawa, H. Hashimoto, S. Koshihara, J. Phys., Conf. Series 148, (2009).

[12] Photoinduced Phase Transitions, Ed. K. Nasu, World Sci., Singapore 2004.

[13] Photo-Induced Phase Transitions and Their Dynamics, Eds. M. Kuwata-Gonokami, S. Koshihara, Special Topics, J. Phys. Soc. Jpn. 75, 011001 (2006).

[14] Dynamical Structural Science, Ed. E. Collet, Special Issue Acta Crystallogr. A 66, 133 (2010).

[15] K.L. Yeh, M.C Hoffmann, J. Hebling, K. Nelson, Appl. Phys. Lett. 90, 171121 (2007).

[16] H. Nakaya, K. Itoh, Y. Takahashi, K. Itoh, S. Iwai, S. Saito, K. Yamamoto, K. Yakushi, Phys. Rev. Lett. 81, 155111 (2010).

[17] K. Tanaka, H. Hihori, M. Nagai, IEEE Trans. Terahertz Sci. Techn. 1, 301 (2011).

[18] G. Fleming, M. Ratner, Physics Today 61, 28 (2008).

[19] A. Zewail, Femtochemistry: Ultrafast Dynamics of the Chemical Bond, Vols. 1 and 2, World Sci., Singapore 1994.

[20] B.C. Gibbs, Nature Chem. 3, 3 (2011).

[21] P.W. Anderson, Science 177, 393 (1972).

[22] Editorial, Nature Phys. 7, 663 (2011).

[23] K. Sokolowski-Titen, C. Blome, J. Blums, A. Cavalleri, c. Dietrich, A. Tarasevitch, I. Uschmann, E. Fôrster, M. Kammler, M. Horn-von Hoegen, D. von der Linde, Nature 422, 287 (2003).

[24] K. Ishida, K. Nasu, Phys. Rev. Lett. 100, 116403 (2008).

[25] L.D. Landau, E.M. Lifshitz, Statistical Physics, Part 1, Vol. 5 of Course of Theoretical Physics, Pergamon Press, Oxford 1980.

[26] D. Chernyshov, H. Bürgi, M. Hostettler, K.W. Törnroos, Phys. Rev. B 70, 094116 (2004).

[27] P.M. Chaikin, T.C. Lubenski, Principles of Condensed Matter Physics, Cambridge University Press, Cambridge 1995.

[28] R. Zeyher, W. Finger, Phys. Rev. Lett. 49, 1833 (1982).

[29] R.D. Averitt, Nature Phys. 6, 639 (2010).

[30] R. Yusupov, T. Mertelj, V.V. Kabanov, S. Brazovkii, P. Kusar, J. Chu, I.R. Fisher, D. Mihailovic, Nature Phys. 6, 681 (2010).

[31] A. Bousseksou, G. Molnar, L. Salmon, W. Nicolazzi, Chem. Soc. Rev. 40, 3313 (2011).

[32] O. Sato, T. Iyoda, A. Fukushima, K. Hashimoto, Science 272, 704 (1996).

[33] S. Ohkoshi, Y. Tsunobuchi, T. Matsuda, K. Hashimoto, A. Namai, F. Hakoe, H. Tokoro, Nature Chem. 2, 539 (2010).

[34] M. Sollogoub, S. Guieu, M. Geoffroy, A. Yamada, A. Estevez-Torres, K. Yoshikawa, D. Baigl, Chem. Biol. Chem. 9, 1201 (2008).

[35] S.H. Stroglatz, Nonlinear Dynamics and Chaos, Perseus Books, Westview 2000. 
[36] A. Hauser, Chem. Phys. Lett. 192, 65 (1992).

[37] K. Koshino, T. Ogawa, J. Phys. Soc. Jpn. 68, 2164 (1999).

[38] K. Boukheddaden, I. Shteto, B. Höo, F. Varret, Phys. Rev. B 62, 14806 (2000).

[39] S. Ohkoshi, K. Imoto, Y. Tsunobuchi, S. Takano, H. Tokoro, Nature Chem. 3, 564 (2011).

[40] N. Bruefel, H. Watanabe, L. Toupet, J. Come, N. Matsumoto, E. Collet, K. Tanaka, J. Tuchagues, Angew. Chem. Int. Ed. 48, 9304 (2009).

[41] R.P. Feynman, Statistical Mechanics, Perseus Books, Westview 1972

[42] H. Cailleau, M. Lorenc, L. Guerin, M. Servol, E. Collet, M. Buron-Le Cointe, Acta Crystallogr. A 66, 189 (2010).

[43] R.G. Palmer, Adv. Phys. 31, 669 (1982).

[44] M. Kurzynski, The Thermodynamic Machinery of Life, Springer, 2006.

[45] M. Cross, H. Greenside, Pattern Formation and Dynamics in Nonequilibrium Systems, Cambridge University Press, Cambridge 2009.

[46] R.C. Desai, R. Kapral, Dynamics of Self-Organized and Self-Assembled Structures, Cambridge University Press, Cambridge 2009.

[47] C. Chong, F. Varret, K. Boukheddaden, Phys. Rev. Lett. 81, 014104 (2010).

[48] K. Onda, S. Ogihara, K. Yonemitsu, N. Maeshima, T. Ishikawa, Y. Okimoto, X. Shao, Y. Nakano, H. Yamochi, G. Saito, S. Koshihara, Phys. Rev. Lett. 101, 067403 (2008)

[49] H. Ichikawa, S. Nozawa, T. Sato, A. Tomita, K. Ichianagi, M. Chollet, L. Guerin, N. Dean, A. Cavalleri, S. Adachi, T. Arima, H. Sawa, Y. Ogimoto, M. Nakamura, R. Tamaki, K. Miyano, S. Koshihara, Nature Mater. 10, 101 (2011).

[50] L. Guerin, J. Hebert, M. Buron-Le Cointe, S. Adachi, S. Koshihara, H. Cailleau, E. Collet, Phys. Rev. Lett. 105, 246101 (2010)

[51] K. Koshino, T. Ogawa, J. Phys. Soc. Jpn. 67, 2174 (1998).

[52] H. Okamoto, Y. Ishige, S. Tanaka, H. Kishida, S. Iwai, Y. Tokura, Phys. Rev. B 70, 165202 (2004).

[53] S. Iwai, Y. Ishige, S. Tanakaz, Y. Okimoto, Y. Tokura, H. Okamoto, Phys. Rev. Lett. 96, 057403 (2006).

[54] S. Koshihara, Y. Takahashi, H. Sakai, Y. Tokura, T. Luty, J. Phys. Chem. B 103, 2592 (1999).

[55] T. Susuki, T. Sakamaki, K. Tanimura, S. Koshihara, Y. Tokura, Phys. Rev. B 60, 6191 (1999).

[56] M. Lorenc, J. Hebert, N. Moisan, E. Trzop, M. Servol, M. Buron-Le Cointe, H. Cailleau, M.L. Boillot, E. Pontecorvo, M. Wulff, S. Kohihara, E. Collet, Phys. Rev. Lett. 103, 028301 (2009)

[57] D. Fausti, R.I. Tobey, N.dean, S. Kaiser, A. Dienst, M.C. Hoffmann, S. Pyon, T. Takayama, H. Takagi, A. Cavalleri, Science 331, 189 (2011).

[58] P. Beaud, S.L. Johson, E. Vorobeva, U. Staub, R.A. De Souza, C.J. Milne, Q.X. Jia, G. Ingold, Phys. Rev. Lett. 103, 155702 (2009).

[59] E. Möhr-Vorobeva, S.L. Johnson, P. Beaud, U. Staub, R. De Souza, C. Milne, G. Ingold, J. Demsar, H. Schaefer, A. Titov, Phys. Rev. Lett. 107, 036403 (2011).
[60] A. Tomeljak, H. Schäfer, D. Städer, M. Beyer, K. Biljakovic, J. Demsar, Phys. Rev. Lett. 102, 066404 (2009).

[61] H. Schäfer, V.V. Kabanov, M. Beyer, K. Biljakovic, J. Demsar, Phys. Rev. Lett. 105, 066402 (2010).

[62] G. Sciaini, M. Hab, S.G. Kruglik, T. Prayer, C.T. Hebeisen, F. Meyer zu Heringdorf, M. Yamaguchi, M. Horn-von Hoegen, R. Ernstorfer, R.J. Dwayne Miller, Nature 458, 56 (2009).

[63] M. Eichberger, H. Schäfer, M. Krumova, M. Beyer, J. Demsar, H. Berger, G. Moriena, G. Sciaini, R.J. Dwayne Miller, Nature 468, 799 (2010).

[64] K. Yamamoto, S. Iwai, S. Boyko, A. Kashiwazaki, F. Hiramatsu, C. Okabe, N. Nishi, K. Yakushi, J. Phys. Soc. Jpn. 77, 074709 (2008).

[65] Y. Kawkami, T. Fukatsu, Y. Sakurai, H. Unno, H. Itoh, S. Iway, T. Sasaki, K. Yamamoto, K. Yakushi, K. Yonemitsu, Phys. Rev. Lett. 105, 246402 (2010).

[66] H. Uemura, H. Okamoto, Phys. Rev. Lett. 105, 258302 (2010).

[67] K. Iwano, Phys. Rev. Lett. 102, 106405 (2009).

[68] Y. Giret, A. Gellé, B. Arnaud, Phys. Rev. Lett. 106, 155503 (2011).

[69] D.M. Fritz, D.A. Reis, B. Adams, R.A. Akre, J. Arthur, C. Blome, P.H. Bucksbaum, A.L. Cavalieri, S. Engemann, S. Fahy, R.W. Falcone, P.H. Fuoss, K.J. Gaffney, M.J. George, J. Hajdu, M.P. Hertlein, P.B. Hillyard, M. Horn-von Hoegen, M. Kammler, J. Kaspar, R. Kienberger, P. Krejcik, S.H. Lee, A.M. Lindenberg, B. McFarland, D. Meyer, T. Montagne, E.D. Murrau, A.J. Nelson, M. Nicoul, R. Pahl, J. Rudati, H. Schlarb, D.P. Siddons, K. Sokolowski-Tinten, Th. Tschentscher, D. von der Linde, J.B. Hastings, Science 315, 633 (2007).

[70] W. Lu, M. Nicoul, U. Shymanovich, A. Tarasevitch, M. Kammler, M. Horn, D. von Hoegen, von der Linde, K. Sokolowski-Tinten, in: Ultrafast Phenomena XVII, Eds. M. Chergui, D. Jonas, E. Riedle, R. Schoenlein, A. Taylor, Oxford University Press, Oxford 2011, p. 314 .

[71] P. Baum, D. Yang, A.H. Zewail, Science 318, 788 (2007).

[72] Y. Okimoto, X. Peng, M. Tamura, T. Morita, K. Onda, T. Ishikawa, S. Koshihara, N. Todokori, T. Kyomen, M. Itoh, Phys. Rev. Lett. 103, 027402 (2009).

[73] Y. Okimoto, M. Kurashima, K. Seko, T. Ishikawa K. Onda, S. Koshihara, T. Kyomen, M. Itoh, Phys. Rev. B 83, 161101 (2011).

[74] O. Fouche, J. Degert, G. Jonusauskas, N. Daro, J.-F. Letard, E. Freysz, Phys. Chem. Chem. Phys. 12, 3044 (2010).

[75] A. Kiriliyuk, A.V. Kimel, T. Rasing, Rev. Mod. Phys. 82, 2731 (2010).

[76] K. Kamide, T. Ogawa, Phys. Rev. Lett. 105, 056401 (2010). 